\title{
Advances in ovarian cancer, from biology to treatment
}

\author{
Recent preclinical and clinical research has led to exciting advances related to high-grade serous ovarian cancer, \\ from examining its cellular origins to gaining insight into DNA-damage-repair mechanisms that may be leveraged \\ for therapies. Furthermore, studies have demonstrated clinical benefit for inhibition of the polymerase PARP and \\ modulation of the cell cycle, and have identified molecular features related to therapeutic response.
}

\section{Michelle McMullen, Katherine Karakasis, Robert Rottapel and Amit M. Oza}

n 2020, the COVID-19 pandemic dominated the global consciousness, directing health policy and research efforts. For those whose lives are touched by ovarian cancer (OC), however, it is important to remember that every year there are over 295,000 new diagnoses of OC and over 184,000 deaths globally ${ }^{1}$. Clinical care and research have been forced to adapt in response to the pandemic and, encouragingly, research into OC has maintained momentum. The adoption of translational endpoints in early-phase trial settings, supported by preclinical discovery, has helped set the stage for the integration of comprehensive translational endpoints into phase 2 trials. Here we take stock of noteworthy advances in OC research from the laboratory to the clinic, establishing important foundations and key research priorities for the road ahead (Fig. 1).

\section{Preclinical developments}

An enduring question in OC research that is playing out in the preclinical arena surrounds the cell of origin for high-grade serous ovarian cancer (HGSOC). A dualistic cell-of-origin model partially supports the notion that there is some biological heterogeneity in HGSOC and would imply that the cell of origin should be considered in the development of therapeutic strategies, as it may critically influence treatment response. A recent study used lineage-specific Cre recombinaseexpressing lines and organoids derived from fallopian tube epithelium and ovarian surface epithelium to generate tumors that emulate human HGSOC in mice, and the results suggest that human HGSOC arises from both the fallopian tube epithelium and ovarian surface epithelium, consistent with a dualistic cell-of-origin model ${ }^{2}$. Another study used CRISPR-Cas9 technology to knock out the tumor suppressor-encoding genes Trp53, Brca1, Nf1 and Pten in mouse cells of either ovarian or oviductal origin and showed that both epithelia can give rise to ovarian tumors ${ }^{3}$. Although both tissue types give rise to ovarian tumors with high-grade pathology, the mutant oviduct organoids expand faster and with higher malignant potential when transplanted ${ }^{3}$. Notably, both of these studies ${ }^{2,3}$ showcased distinct lineage-dependent sensitivities to therapies that are commonly used to treat HGSOC, including inhibitors of poly(ADP-ribose) polymerase (PARPi), and paclitaxel and carboplatin ${ }^{2,3}$. These observations remain to be validated in human models of HGSOC, but they may eventually inform the development of more-precise treatment strategies based on the cell of origin for a patient's tumor and relative chemosensitivites ${ }^{3}$.

The genomes of HGSOC tumors are typified by widespread damage and copy-number alteration, and the response to DNA damage underlies the ability of these tumors to manage genotoxic stress ${ }^{4}$. Ongoing work spanning the preclinical and clinical space is aimed to improve understanding of DNA-damage-repair mechanisms and the processes involved in safeguarding cell fidelity that may be leveraged in therapeutic development. A study using CRISPR-Cas9 to generate a functional genetic map of DNA damage responses to 27 genotoxic agents in retinal pigment epithelial cells has created a rich resource to study this fundamental cellular system, with implications for the development and use of genotoxic agents in OC therapy. These comprehensive screens identified 890 genes whose loss contributes to either sensitivity or resistance to DNA-damaging agents ${ }^{5}$. They showed that pyridostatin, which induces guanine (G) quadruplexes, induces DNA damage and cell-cycle arrest through a process that involves the trapping of topoisomerase II on $\mathrm{DNA}^{5}$, a concept that will be explored in upcoming trials of G-quadruplex stabilization in OC.

\section{Phase $1 / 2$ clinical trials}

In early-phase clinical drug development, overcoming therapeutic resistance remains a major challenge. A broad portfolio of trials are ongoing to assess rational therapeutic combinations directed at OC tumors and their microenvironment, including chemotherapies, targeted therapies and immunotherapies. For example, the TOPACIO/KEYNOTE-162 phase $1 / 2$ trial of niraparib and pembrolizumab in recurrent OC (NCT02657889) reported an overall objective response rate of $25 \%$ and an objective response rate of $45 \%$ in patients with BRCA-mutated (BRCAm) OC. Immunogenomic profiling of the 62 patients revealed that presence of the mutational signature designated ' 3 ', reflective of defective homologous recombination DNA repair, and a positive immune score for gene expression related to interferon signaling and exhausted $\mathrm{CD} 8^{+} \mathrm{T}$ cells in the tumor microenvironment, were both associated with response to the combination therapy ${ }^{6}$. Highly multiplexed single-cell imaging of tumor samples suggested that interactions of exhausted CD8 ${ }^{+} \mathrm{T}$ cells with either macrophages or tumor cells that expressed the the checkpoint ligand PD-L1 were mechanistic determinants of response $\mathrm{e}^{6}$. Such integration of translational endpoints in these clinical trials accelerates feedback into basic research by highlighting response mechanisms that can be taken back to the laboratory to guide future research. Specifically, these findings lend support to the hypothesis that DNA damage mediated by PARPi modulates the tumor immune microenvironment, which suggests that the combination of PARPi plus immunotherapy represents a rational combination strategy for OC, and provides mechanistic insight into biomarkers that may be incorporated into future immunotherapy trials in OC.

Cell-cycle modulation has been pursued for OC treatment, as cell-cycle control mechanisms represent a critical component 

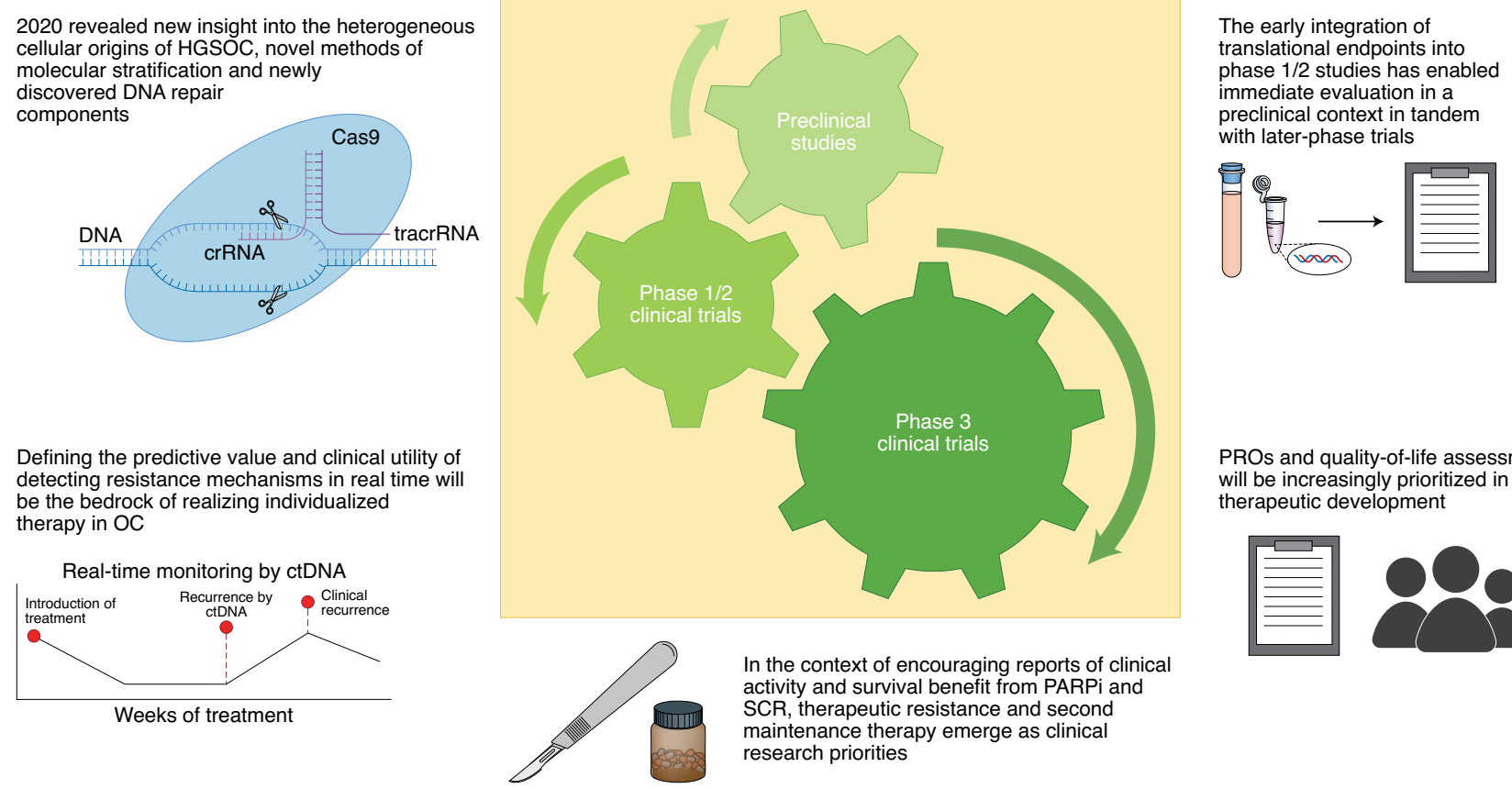

PROs and quality-of-life assessments will be increasingly prioritized in therapeutic development
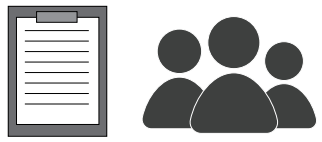

Fig. 1 | Key research achievements and emerging priorities in 2020. Activities completed over the course of 2020 have advanced the understanding of OC in the laboratory and the clinic. The adoption of translational endpoints in early-phase trial settings, supported by preclinical discovery, has helped set the

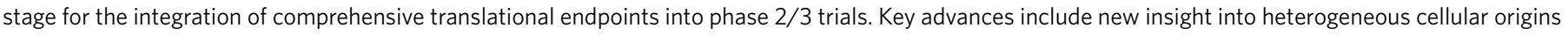
of HGSOC and therapeutic opportunities in newly identified components of DNA repair, and clinical benefit demonstrated from new approaches, including

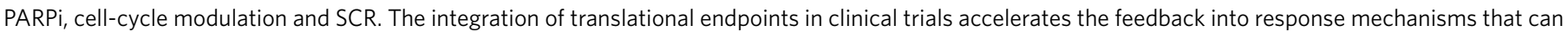

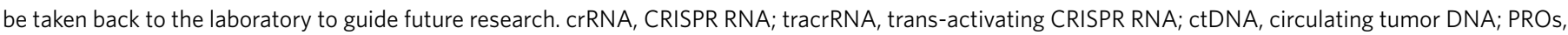
patient-reported outcomes. Figure created using BioRender.com.

of the DNA-damage response, able to effectively detect DNA damage and halt cellular replication in order to initiate repair. Adavosertib (AZD1775), which inhibits the nuclear kinase WEE1, has emerged as a promising compound that is able to regulate $\mathrm{G}_{2}-\mathrm{M}$ transition and sensitize tumor suppressor p53-mutant cells to chemotherapy ${ }^{7}$. This year, the results of a double-blind phase 2 trial (NCT01357161) were reported in which 141 women with recurrent platinum-sensitive $\mathrm{OC}$ in which TP53 is mutated were randomized to receive oral adavosertib or placebo, in addition to chemotherapy ${ }^{7}$. The addition of adavosertib improved progression-free survival (PFS) according to the enhanced RECIST 1.1 guidelines (ePFS; hazard ratio (HR), 0.63; 95\% confidence interval (CI) 0.38-1.06; two-sided $P=0.080)$, meeting the predefined significance threshold $(P<0.2)^{7}$. Clinical benefit was broadly observed for patients with a variety of different subtypes of TP53 mutation, which identified potential response biomarkers ${ }^{7}$. The clinical potential of adavosertib gained momentum following early results of two other studies. Results from a randomized phase 2 trial assessing adavosertib in combination with gemcitabine in platinum-resistant OC (NCT02151292) $)^{8}$ demonstrated benefits to PFS (HR, 0.56; 95\% CI, 0.35-0.90; $P=0.015$, log-rank test) and overall survival (OS) (HR, 0.56; 95\% CI, 0.34-0.92; $P=0.022)^{8}$. Another group reported remarkable activity of adavosertib monotherapy in recurrent serous endometrial cancer, demonstrating an objective response rate of $29.4 \%$ (95\% CI, 15.1-47.5\%) in a two-stage single-arm phase 2 study (NCT03668340) ${ }^{9}$ that enrolled 35 patients. Together these studies expand the complex picture of cellular replication stress, pointing beyond inhibition of WEE1 to solidify the use of strategies involving inhibition of DNA-damage response and modulation of the cell cycle in OC, and thereby create opportunities for refining treatment strategies in the setting of advanced disease.

\section{Phase 3 clinical trials}

PARPi have continued to dominate the landscape of practice-changing randomized clinical trials. Recent seminal trials (PAOLA 1 (NCT02477644), VELIA (NCT02470585) and PRIMA (NCT02655016)) demonstrated significant improvements in PFS driven by the use of PARPi as maintenance therapy while also establishing the potential of using predictive biomarker signatures such as homologous-recombination deficiency (HRD) to identify patients who would not benefit from treatment with PARPi. Important differences exist between the trials in terms of the clinical characteristics of patients enrolled in each, including the use of different companion diagnostics to define HRD positivity, and these nuanced details should be considered in the selection of the appropriate treatment with PARPi. In the PAOLA 1 trial (NCT02477644), the addition of maintenance olaparib to standard first-line therapy, including bevacizumab, resulted in significant PFS benefit compared with PFS after treatment with bevacizumab and placebo. The effect was most evident in patients with HRD-positive tumors (defined as an HRD score of $\geq 42$, by the Myriad Genetic Laboratories myChoice HRD Plus assay; median PFS, 37.2 months versus 17.7 months), including those without BRCAm (median PFS, 28.1 months versus 16.6 months). In the VELIA trial (NCT02470585), the addition of veliparib to standard front-line platinum chemotherapy followed by maintenance veliparib 
resulted in significant improvement in PFS compared with the PFS of those who received placebo, as analyzed sequentially in a BRCAm cohort (median PFS, 34.7 months versus 22 months); an HRD-positive cohort that included patients with BRCAm (31.9 months versus 20.5 months); and an 'intention-to-treat' population (23.5 months versus 17.3 months). The PRIMA study (NCT02655016) found significant improvements in median PFS with niraparib maintenance treatment after a response to front-line platinum chemotherapy, with hierarchical testing demonstrating benefit in an HRD-positive population (21.9 months versus 10.4 months) and 'all comers' (13.8 months versus 8.2 months). The updated analysis of the SOLO 2 trial (NCT01874353) was notable in showing that maintenance olaparib in women with BRCAm recurrent OC led to an improvement in median OS compared with the median OS of those who received placebo (52 months for olaparib versus 39 months for placebo; $H R, 0.74$; $95 \%$ CI, 0.54-1.00; $P=0.053)^{10}$. In patients newly diagnosed with advanced OC, the 5 -year follow up data from the SOLO 1 trial (NCT01844986) ${ }^{11}$ demonstrated sustained benefit derived from 2 years of maintenance olaparib in patients with BRCAm: $48.3 \%$ of those who received olaparib remained progression free at 5 years, compared with $20 \%$ of patients who received placebo ${ }^{11}$. This follow-up study also established that there was a significant incidence of myelodysplastic syndrome in patients treated with PARPi ( $8 \%$ at 5 years in SOLO1), which reinforces the need for careful monitoring. Now that PARPi are established as maintenance therapies in the front-line and recurrence settings, the role of maintenance via 'PARPi after PARPi' remains an unanswered question. This will be explored in the ongoing randomized phase $3 \mathrm{~b}$ OReO trial assessing olaparib maintenance therapy versus placebo in relapsed, platinum-sensitive OC previously treated with PARPi (NCT03106987). Collaborative groups now seek to define approaches for the setting after the use of PARPi. A coordinated approach to translational studies that elucidates mechanisms of resistance to PARPi and predictive biomarkers may also help tailor subsequent therapies and their sequence.

Two randomized phase 3 trials in recurrent platinum-sensitive OC affirmed the role of secondary cytoreductive surgery (SCR). The AGO DESKTOP III/ENGOT-ov20 trial (NCT01166737) $)^{12}$ enrolled 407 patients with recurrent platinum-sensitive $\mathrm{OC}$ and a positive AGO score (as determined by an ECOG status of 0 , an ascites volume of $\leq 500 \mathrm{ml}$ and complete resection (R0) at initial surgery) who were then prospectively randomized to receive either second-line chemotherapy or SCR followed by chemotherapy ${ }^{12}$. SCR led to a meaningful improvement in OS (53.7 months with surgery and 46.2 months without surgery; HR, 0.76; 95\% CI, $0.59-0.97 ; P=0.03$ ) exclusively among those patients in whom complete resection was achieved. The SOC1/SGOG-OV2 trial $^{13}$ used the iMODEL score combined with PET-CT to select patients predicted to undergo R0 resection, and demonstrated that SCR and chemotherapy improved PFS relative to the PFS after chemotherapy alone (PFS, 17.4 months versus 11.9 months; HR, 0.58; 95\% CI, 0.45-0.74; $P<0.001)$. These results are a departure from those of an earlier randomized phase 3 trial, GOG-0213 (NCT00565851) $)^{14}$, which did not find that SCR followed by chemotherapy improved OS relative to OS after chemotherapy alone (HR, 1.29; 95\% CI, 0.97-1.72; $P=0.08)$ ). Together these trials suggest that clinical benefit from SCR is dependent on achievement of $\mathrm{R} 0$ and that the effect may be mitigated by systemic therapy such as bevacizumab; they also demonstrate the value of implementing careful patient-selection methods, such as the AGO and iMODEL tools used here. The roles of neoadjuvant and adjuvant therapy with PARPi after SCR will be explored by the ongoing randomized phase 2 NEO trial (NCT02489006).

\section{Conclusions}

This past year has seen many foundational advances in OC, with important new insights pointing toward heterogeneous cellular origins of HGSOC and newly identified therapeutic opportunities in components of the DNA-repair pathway. Improved understanding of this biological complexity feeds future translational strategies for improving therapeutic precision and leveraging molecular vulnerabilities in individual cases. Researchers continue to gain insight into the clinical activity of new therapeutic approaches such as PARPi and cell-cycle modulation, as well as the molecular features related to therapeutic benefits. We expect to see broader uptake of SCR in carefully selected patients in future, on the basis of the tools and criteria established in the seminal SCR trials. With increasing uptake of maintenance via PARPi, therapeutic resistance and the role of second maintenance treatment emerge as key clinical research priorities. At the center of each discovery remain the women with OC. Results from the World Ovarian Cancer Coalition's Every Woman Study ${ }^{15}$, obtained through surveys and interviews from 1,531 women in 44 countries, highlighted significant regional variation in delays to diagnosis and access to specialist treatment, which translate into differences in clinical outcomes. In a time in which issues of racial and societal equity have also been brought powerfully to the forefront of our global consciousness, this reinforces the need to place the powerful voices of patients at the forefront of the scientific enquiry, and encourages clinical trials to integrate patient-reported outcomes and quality-of-life assessments into therapeutic development. Looking forward, defining the predictive value and clinical utility of detecting resistance mechanisms in 'real time', and clinically validating liquid biopsy and other molecular stratification tools aimed at increasing the speed, efficiency and accuracy of predictive biomarker analysis, will bring the field closer to a cost-effective and, notably, patient-centric reality.

\section{Michelle McMullen', Katherine Karakasis', Robert Rottape $\mathrm{I}^{2,3,4,5,6}$ and Amit M. Oza ${ }^{1 凶}$ ${ }^{1}$ Division of Medical Oncology \& Hematology, Bras Family Drug Development Program, Princess Margaret Cancer Centre, University Health Network, Toronto, Ontario, Canada. ${ }^{2}$ Princess Margaret Cancer Centre, University Health Network, Toronto, Ontario, Canada. ${ }^{3}$ Department of Medical Biophysics, University of Toronto, Toronto, Ontario, Canada. ${ }^{4}$ Department of Medicine, University of Toronto, Toronto, Ontario, Canada. ${ }^{5}$ Department of Immunology, University of Toronto, Toronto, Ontario, Canada. ${ }^{6}$ Division of Rheumatology, St. Michael's \\ Hospital, Toronto, Ontario, Canada.

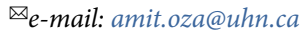

Published online: 13 January 2021 https://doi.org/10.1038/s43018-020-00166-5

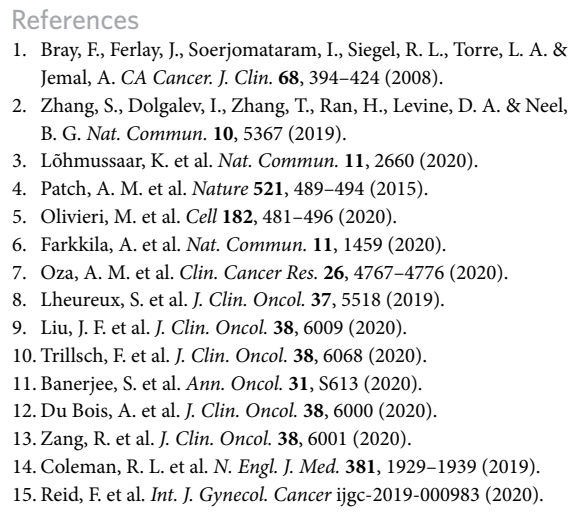

Competing interests

A.M.O. declares grants to his institution from AstraZeneca, outside the submitted work, and membership on uncompensated steering committees with AstraZeneca and Clovis and uncompensated advisory role with AstraZeneca and GSK. A.M.O. is principal investigator on investigator-initiated trials with agents from AstraZeneca, GSK and Clovis. 